\title{
Sliding Mode Control for NSVs with Input Constraint Using Neural Network and Disturbance Observer
}

\author{
Yan-long Zhou and Mou Chen \\ College of Automation Engineering, Nanjing Aeronautic and Astronautic University, Nanjing 210016, China \\ Correspondence should be addressed to Mou Chen; chenmou@nuaa.edu.cn
}

Received 10 May 2013; Accepted 15 July 2013

Academic Editor: William Guo

Copyright (c) 2013 Y.-1. Zhou and M. Chen. This is an open access article distributed under the Creative Commons Attribution License, which permits unrestricted use, distribution, and reproduction in any medium, provided the original work is properly cited.

\begin{abstract}
The sliding mode control (SMC) scheme is proposed for near space vehicles (NSVs) with strong nonlinearity, high coupling, parameter uncertainty, and unknown time-varying disturbance based on radial basis function neural networks (RBFNNs) and the nonlinear disturbance observer (NDO). Considering saturation characteristic of rudders, RBFNNs are constructed as a compensator to overcome the saturation nonlinearity. The stability of the closed-loop system is proved, and the tracking error as well as the disturbance observer error can converge to the origin through the Lyapunov analysis. Simulation results are presented to demonstrate the effectiveness of the proposed flight control scheme.
\end{abstract}

\section{Introduction}

The NSV [1-3] is a kind of vehicles and flights above $20 \mathrm{~km}$ from the ground, which can be divided into low-speed NSV and high-speed NSV. Among them, the high-speed NSV has some significant advantages such as rapid response ability, difficulty of detecting, and strong penetration ability. Thus, it has drawn much attention around the world in recent years. Since the NSV has the large flight envelop as well as the changeable flight environment and it is susceptible to the severely external time-varying disturbance, it is necessary to develop the robust control scheme for the NSV. However, strong nonlinearity, high coupling, parameter uncertainty, and unknown time-varying disturbance will further increase the design difficulty of the robust flight control [4]. To efficiently handle the system uncertainty and the suffering unknown external disturbance, NDO can be employed to design the robust control scheme. According to the known information of the studied system, NDO can estimate the compounded disturbance on line and feedback the estimate value to the control system. As a result, the disturbance cancellation is guaranteed to improve the performance and robustness of the closed-loop system. Therefore, the technology of NDO has been extensively studied. In [4-7], NDO was used to estimate the system uncertainty and the unknown external disturbance, and it was successfully applied to the robust control design of inverted pendulum and air vehicles, respectively.

Saturation as a common input nonlinearity exists in a wide range of practical systems, such as the NSV. The existence of input saturation will degrade the control system performance and even leads to the system instability if it is ignored in the control design. On the other hand, the control design considering input saturation is a challenging problem for uncertain nonlinear systems. Several control schemes for nonlinear systems with input saturation have been proposed in recent years. In [8-10], neural network was proposed to approximate the input-output difference of the actuator, and a compensator was designed to overcome the saturation nonlinearity. In [11, 12], considering the smoothness of a hyperbolic tangent function, the robust adaptive control was proposed based on the backstepping approach via using the special property of a Nussbaum function to handle input saturation. However, the rudder saturation of the NSV needs to be further studied in the robust attitude control design.

For the nonlinear systems, many robust control schemes were studied [13-18] in which SMC is an effective method. By applying a discontinuous control signal to change the system state, the system is forced to slide along a predesigned sliding mode. It has higher robustness because of the insensitivity to 
the parameter uncertainty. However, the control input needs to be changed according to the system states, which causes the system trajectory passing across the sliding mode repeatedly. Thus, there exists chattering problem in the system [19]. By designing a new switching function which is related to the first-order or higher-order derivative of the control input, the continuous dynamic sliding mode control law can be obtained though integration. Therefore, the system chattering can be effectively reduced. In [20], a robust dynamic sliding mode control law was proposed to control a multi-degreeof-freedom humanoid robot arm, and satisfactory control results had been obtained through simulation. In [21], higherorder sliding mode control and sliding mode differentiator were studied. In [22], a second-order dynamic sliding control scheme was proposed to overcome chattering problem for the NSV. However, the sliding mode control considering input saturation should be further developed for the NSV based on NDO.

Motivated by above discussion and analysis, the robust attitude control is developed for the NSV in the presence of the system uncertainty, the unknown time-varying disturbance, and the input saturation. To handle the compounded disturbance, the NDO is proposed. Since the rudders of the NSV have the saturation characteristic, RBFNNs are constructed as a compensator to overcome the saturation nonlinearity. The stability of the closed-loop system is proved, and the tracking error as well as the disturbance observer error can converge to the origin through the Lyapunov analysis. The organization of the paper is as follows. In Section 2, the dynamic sliding mode control is reviewed. The robust attitude control is investigated for the NSV by considering the system uncertainty, the unknown external disturbance, and the input saturation in Section 3, and simulation results are given to illustrate the effectiveness of the proposed robust attitude control scheme. Section 4 concludes the remark.

For the convenience of description, the following notations are required.

Notations. Throughout this paper, $|\bullet|$ stands for absolute value of each element of the vector; $|\cdot|^{c}$ denotes power operation for the absolute value of each element of the vector, where $c$ is a power exponent; $\operatorname{sgn}(\bullet)$ stands for sign function operation of each element of the vector; $\|\bullet\|$ represents the Euclidean norm (or Frobenius norm for a matrix); $\int_{0}^{t}(\bullet) d t$ stands for integral operation of each element of the vector; $\operatorname{diag}(\bullet)$ represents a diagonal matrix constructed by the elements of the vector; $\operatorname{diag}\{\operatorname{sgn}(\bullet)\}$ denotes constructing a diagonal matrix after sign function operation of the vector. Specifically, given a matrix $W$ and a vector $\chi$, then we have

$$
\begin{gathered}
|\chi|=\left[\left|\chi_{1}\right|,\left|\chi_{2}\right|, \ldots,\left|\chi_{n}\right|\right]^{T}, \\
|\chi|^{c}=\left[\left|\chi_{1}\right|^{c},\left|\chi_{2}\right|^{c}, \ldots,\left|\chi_{n}\right|^{c}\right]^{T}, \\
\operatorname{sgn}(\chi)=\left[\operatorname{sgn}\left(\chi_{1}\right), \operatorname{sgn}\left(\chi_{2}\right), \ldots, \operatorname{sgn}\left(\chi_{n}\right)\right]^{T}, \\
\|\chi\|=\sqrt{\chi_{1}^{2}+\chi_{2}^{2}+\cdots+\chi_{n}^{2}},
\end{gathered}
$$

$$
\begin{gathered}
\|W\|=\sqrt{\sum_{i=1}^{n} \sum_{j=1}^{m} w_{i j}^{2}, W \in R^{n \times m},} \\
\int_{0}^{t} \chi d t=\left[\int_{0}^{t} \chi_{1} d t, \int_{0}^{t} \chi_{2} d t, \ldots, \int_{0}^{t} \chi_{n} d t\right]^{T}, \\
\operatorname{diag}(\chi)=\left[\begin{array}{ccc}
\chi_{1} & \cdots & 0 \\
\vdots & \ddots & \vdots \\
0 & \cdots & \chi_{n}
\end{array}\right] \in R^{n \times n}, \\
\operatorname{diag}(\operatorname{sgn}(\chi))=\left[\begin{array}{ccc}
\operatorname{sgn}\left(\chi_{1}\right) & \cdots & 0 \\
\vdots & & \ddots \\
0 & \cdots & \operatorname{sgn}\left(\chi_{n}\right)
\end{array}\right] \in R^{n \times n} .
\end{gathered}
$$

\section{Design of Dynamic Sliding Mode Control for MIMO System}

In this paper, the dynamic sliding mode control scheme is used to the robust attitude control design of the NSV. For the convenience of control development, the dynamic sliding mode control scheme is described as follows according to $[22,23]$.

Consider that a class of multiinput and multioutput (MIMO) nonlinear affine systems is given by

$$
\begin{gathered}
\dot{x}(t)=f(x(t))+g(x(t)) u(t), \\
y(t)=h(x(t)),
\end{gathered}
$$

where $x \in R^{n}$ is the state vector of the nonlinear system, $y \in$ $R^{m}$ is the output vector, $u \in R^{m}$ is the control input vector, and $f(x) \in R^{n}, g(x) \in R^{n \times m}$, and $h(x) \in R^{m}$ are continuous functions which are related to the state vector $x$. Suppose that the sliding mode surface for the MIMO nonlinear system (2) can be written as $\sigma=\left[\sigma_{1}(x), \sigma_{2}(x), \ldots, \sigma_{m}(x)\right]^{T} \in R^{m}$.

To analyze the nonlinear system (2), the following definitions and assumptions are required.

Definition 1 (see [24]). The gradient of the smooth scalar function $\sigma_{i}(x)$ with respect to the state vector $x$ is defined as

$$
\nabla \sigma_{i}(x)=\frac{\partial \sigma_{i}(x)}{\partial x}=\left[\frac{\partial \sigma_{i}(x)}{\partial x_{1}}, \frac{\partial \sigma_{i}(x)}{\partial x_{2}}, \ldots, \frac{\partial \sigma_{i}(x)}{\partial x_{n}}\right] .
$$

Definition 2 (see [24]). The Lie derivative of the smooth scalar function $\sigma_{i}(x)$ with respect to the vector field $f(x)$ is given by

$$
L_{f} \sigma_{i}(x)=\nabla \sigma_{i}(x) f(x) .
$$

The multiple Lie derivative can be defined as the following recurrence relations:

$$
\begin{gathered}
L_{f}^{0} \sigma_{i}(x)=\sigma_{i}(x), \\
L_{f}^{k_{i}} \sigma_{i}(x)=L_{f} L_{f}^{k_{i}-1} \sigma_{i}(x)=\nabla\left(L_{f}^{k_{i}-1} \sigma_{i}(x)\right) f(x), \\
k_{i}=1,2,3, \ldots
\end{gathered}
$$




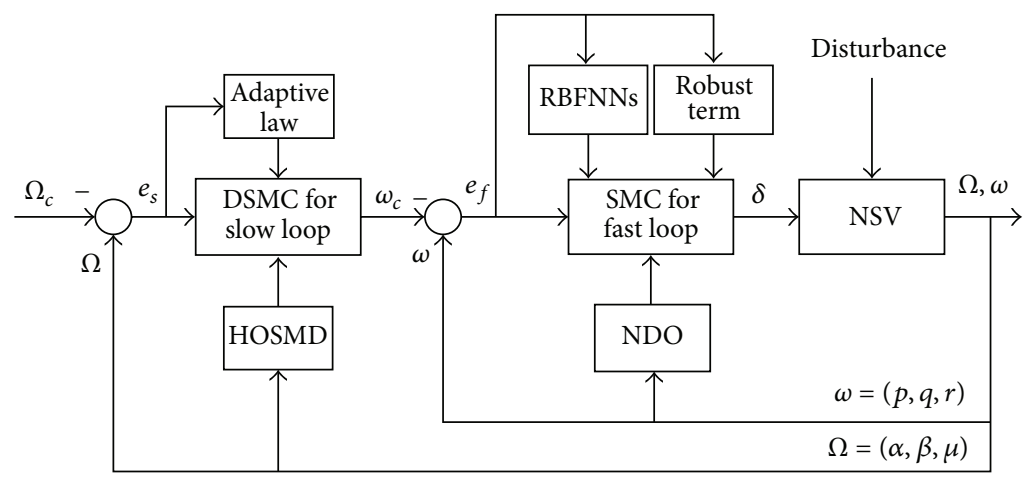

FIGURE 1: structure of the robust control scheme for the NSV.

Definition 3 (see [24]). If the Lie derivative of the smooth scalar function $\sigma_{i}(x)$ with respect to the vector field $f(x)$ satisfies the following two expressions:

$$
\begin{gathered}
L_{f}^{k_{i}} \sigma_{i}(x)=0, \quad k_{i}=0,1, \ldots, r_{i}-1, \\
L_{f}^{r_{i}} \sigma_{i}(x) \neq 0,
\end{gathered}
$$

then the relative degree of $\sigma_{i}(x)$ with respect to $f(x)$ is $r_{i}$. Also, the relative degree of $\sigma=\left[\sigma_{1}(x), \sigma_{2}(x), \ldots, \sigma_{m}(x)\right]^{T}$ with respect to $f(x)$ is $r$, where $r=\max \left\{r_{1}, r_{2}, \ldots, r_{m}\right\}$.

Assumption 4 (see [22]). For the MIMO nonlinear system (2), the state $x$ can converge to the origin when the system enters the sliding mode of $\sigma=0$.

Assumption 5 (see [22]). For the MIMO nonlinear system (2), the relative degree vector of the sliding mode $\sigma$ with respect to the input $u$ is $\left[r_{1}, r_{2}, \ldots, r_{m}\right]$. That is, the following equations are always held for all $x$ in the domain:

$$
\begin{gathered}
\sum_{j=1}^{m} L_{g_{j}} L_{f}^{k_{i}} \sigma_{i}(x)=0, \quad k_{i}=0,1, \ldots, r_{i}-1, \\
\sum_{j=1}^{m} L_{g_{j}} L_{f}^{r_{i}-1} \sigma_{i}(x) \neq 0
\end{gathered}
$$

where $g_{j}$ is the $j$ th column vector of the matrix $g, i=$ $1,2, \ldots, m$.

Assumption 6 (see [22]). For the MIMO nonlinear system (2), the following matrix is invertible for all $x$ in the domain:

$$
\left[\begin{array}{cccc}
L_{g_{1}}\left(L_{f}^{r_{1}-1} \sigma_{1}(x)\right) & L_{g_{2}}\left(L_{f}^{r_{1}-1} \sigma_{1}(x)\right) & \cdots & L_{g_{m}}\left(L_{f}^{r_{1}-1} \sigma_{1}(x)\right) \\
L_{g_{1}}\left(L_{f}^{r_{2}-1} \sigma_{2}(x)\right) & L_{g_{2}}\left(L_{f}^{r_{2}-1} \sigma_{2}(x)\right) & \cdots & L_{g_{m}}\left(L_{f}^{r_{2}-1} \sigma_{2}(x)\right) \\
\vdots & \vdots & \ddots & \vdots \\
L_{g_{1}}\left(L_{f}^{r_{m}-1} \sigma_{m}(x)\right) & L_{g_{2}}\left(L_{f}^{r_{m}-1} \sigma_{m}(x)\right) & \cdots & L_{g_{m}}\left(L_{f}^{r_{m}-1} \sigma_{m}(x)\right)
\end{array}\right]
$$

$$
\in R^{m \times m} \text {. }
$$

The derivative of $\sigma_{i}(x)$ is given by

$$
\begin{aligned}
\sigma_{i}^{\left(k_{i}\right)}= & L_{f}^{k_{i}} \sigma_{i}(x), \quad k_{i}=0,1, \ldots, r_{i}-1, \\
\sigma_{i}^{\left(r_{i}\right)}= & L_{f}^{r_{i}} \sigma_{i}(x)+\sum_{j=1}^{m} L_{g_{j}} L_{f}^{r_{i}-1} \sigma_{i}(x) u_{j}, \\
\sigma_{i}^{\left(r_{i}+1\right)}= & L_{f}^{r_{i}+1} \sigma_{i}(x)+\sum_{j=1}^{m} L_{g_{j}} L_{f}^{r_{i}} \sigma_{i}(x) u_{j} \\
& +\sum_{l=1}^{m} L_{g_{l}}\left(\sum_{j=1}^{m} L_{g_{j}} L_{f}^{r_{i}-1} \sigma_{i}(x) u_{j}\right) u_{l} \\
& +\sum_{j=1}^{m} L_{f} L_{g_{j}} L_{f}^{r_{i}-1} \sigma_{i}(x) u_{j} \\
& +\sum_{j=1}^{m} L_{g_{j}} L_{f}^{r_{i}-1} \sigma_{i}(x) \dot{u}_{j},
\end{aligned}
$$

where $i=1,2, \ldots, m$.

Design a new sliding mode as

$$
\vartheta_{i}=\sigma_{i}^{\left(r_{i}\right)}+c_{i, 1} \sigma_{i}^{\left(r_{i}-1\right)}+\cdots+c_{i, r_{i}-1} \dot{\sigma}_{i}+c_{i, r_{i}} \sigma_{i}+c_{i, r_{i}+1},
$$

where $c_{i, j}\left(i=1,2, \ldots, m, j=1,2, \ldots, r_{i}+1\right)$ must make the polynomial (12) Hurwitz stable.

The derivative of $\dot{\vartheta}_{i}$ is given by

$$
\begin{aligned}
\dot{\vartheta}_{i} & =\sigma_{i}^{\left(r_{i}+1\right)}+c_{i, 1} \sigma_{i}^{\left(r_{i}\right)}+\cdots+c_{i, r_{i}-1} \ddot{\sigma}_{i}+c_{i, r_{i}} \dot{\sigma}_{i} \\
& =\sigma_{i}^{\left(r_{i}+1\right)}+\sum_{j=1}^{r_{i}} c_{i, j} \sigma_{i}^{\left(r_{i}+1-j\right)} .
\end{aligned}
$$

Considering (11) and (13), we obtain

$$
\begin{aligned}
& \dot{\vartheta}=A(x, u)+B(x) \dot{u}+C(x, u), \\
& A(x, u)=A_{1}(x)+A_{2}(x) u+A_{3}(x) u+A_{4}(x) u,
\end{aligned}
$$

where $\dot{\vartheta}=\left[\dot{\vartheta}_{1}, \dot{\vartheta}_{2}, \ldots, \dot{\vartheta}_{m}\right]^{T}$, and we have 


$$
\begin{aligned}
& A_{1}(x)=\left[L_{f}^{r_{1}+1} \sigma_{1}(x), L_{f}^{r_{2}+1} \sigma_{2}(x), \ldots, L_{f}^{r_{m}+1} \sigma_{m}(x)\right]^{T} \in R^{m}, \\
& A_{2}(x)=\left[\begin{array}{ccc}
L_{g_{1}} L_{f}^{r_{1}} \sigma_{1}(x) & \cdots & L_{g_{m}} L_{f}^{r_{1}} \sigma_{1}(x) \\
\vdots & \ddots & \vdots \\
L_{g_{1}} L_{f}^{r_{m}} \sigma_{m}(x) & \cdots & L_{g_{m}} L_{f}^{r_{m}} \sigma_{m}(x)
\end{array}\right] \in R^{m \times m} \\
& A_{3}(x)=\left[\begin{array}{ccc}
L_{f} L_{g_{1}} L_{f}^{r_{1}-1} \sigma_{1}(x) & \cdots & L_{f} L_{g_{m}} L_{f}^{r_{1}-1} \sigma_{1}(x) \\
\vdots & \ddots & \vdots \\
L_{f} L_{g_{1}} L_{f}^{r_{m}-1} \sigma_{m}(x) & \cdots & L_{f} L_{g_{m}} L_{f}^{r_{m}-1} \sigma_{m}(x)
\end{array}\right] \in R^{m \times m} \\
& A_{4}(x)=\left[\begin{array}{ccc}
L_{g_{1}}\left(\sum_{j=1}^{m} L_{g_{j}} L_{f}^{r_{1}-1} \sigma_{1}(x) u_{j}\right) & \cdots & L_{g_{m}}\left(\sum_{j=1}^{m} L_{g_{j}} L_{f}^{r_{1}-1} \sigma_{1}(x) u_{j}\right) \\
\vdots & \ddots & \vdots \\
L_{g_{1}}\left(\sum_{j=1}^{m} L_{g_{j}} L_{f}^{r_{m}-1} \sigma_{m}(x) u_{j}\right) & \cdots & L_{g_{m}}\left(\sum_{j=1}^{m} L_{g_{j}} L_{f}^{r_{m}-1} \sigma_{m}(x) u_{j}\right)
\end{array}\right] \in R^{m \times m}, \\
& B(x)=\left[\begin{array}{ccc}
L_{g_{1}} L_{f}^{r_{1}-1} \sigma_{1}(x) & \cdots & L_{g_{m}} L_{f}^{r_{1}-1} \sigma_{1}(x) \\
\vdots & \ddots & \vdots \\
L_{g_{1}} L_{f}^{r_{m}-1} \sigma_{m}(x) & \cdots & L_{g_{m}} L_{f}^{r_{m}-1} \sigma_{m}(x)
\end{array}\right] \in R^{m \times m} \\
& C(x, u)=\left[\sum_{j=1}^{r_{1}} c_{1, j} \sigma_{1}^{\left(r_{1}+1-j\right)}, \sum_{j=1}^{r_{2}} c_{2, j} \sigma_{2}^{\left(r_{2}+1-j\right)}, \ldots, \sum_{j=1}^{r_{m}} c_{m, j} \sigma_{m}^{\left(r_{m}+1-j\right)}\right]^{T} \in R^{m}
\end{aligned}
$$

To ensure the reaching condition of the sliding mode surface hold, the reaching law $\Phi_{\vartheta}$ can take an exponential form which is described by

$$
\Phi_{\vartheta}=-\bar{C}_{1} \vartheta-\bar{C}_{2} \operatorname{sgn}(\vartheta)
$$

where $\bar{C}_{i}=\operatorname{diag}\left\{\bar{c}_{i, 1}, \bar{c}_{i, 2}, \ldots, \bar{c}_{i, m}\right\}>0, i=1,2$, are designed matrices.

Considering (14) and (16), we have

$$
\dot{u}=B^{-1}(x)\left(\Phi_{\vartheta}-A(x, u)-C(x, u)\right) .
$$

Integration of (17) yields

$$
u(t)=u(0)+\int_{0}^{t}\left(B^{-1}(x)\left(\Phi_{\vartheta}-A(x, u)-C(x, u)\right)\right) d t
$$

Since the discontinuous term of the reaching law is taken into integration, we can obtain a continuous control input in the whole time domain. Thus, the chattering of the system can be effectively reduced.

\section{Design of Robust Attitude Control for the NSV}

3.1. Attitude Dynamic Model for the NSV. Due to the fact that the change of attitude angular rate is faster than that of attitude angle, the attitude motion can be decomposed into two affine MIMO nonlinear systems [25] in accordance with singularly perturbed theory and time-scale separation principle:

$$
\begin{gathered}
\dot{\Omega}=f_{s}(\Omega)+g_{s}(\Omega) \omega+g_{s \delta}(\Omega) \delta(v(t)), \\
y_{s}=\Omega, \\
\dot{\omega}=f_{f}(\omega)+g_{f 1} g_{f \delta}(\omega) \delta(v(t)), \\
y_{f}=\omega,
\end{gathered}
$$

where (19) are called slow-loop equation and fast-loop equation, respectively, and $\Omega=[\alpha, \beta, \mu]^{T}$ is the vector of attitude angles which are angle of attack, sideslip angle, and roll angle, $\omega=[p, q, r]^{T}$ is the vector of attitude angular rates which are roll angular rate, pitch angular rate, and yaw angular rate. In slow-loop equation, $f_{s}(\Omega)=\left[f_{\alpha}, f_{\beta}, f_{\mu}\right]^{T}$ 
is the state function vector, $g_{s}(\Omega)$ is the system matrix, and $g_{s \delta}(\Omega)$ is allocation matrix of rudders. In fast-loop equation, $f_{f}(\omega)=\left[f_{p}, f_{q}, f_{r}\right]^{T}$ is the state function vector, $g_{f 1}$ is the system matrix, $g_{f \delta}(\omega)$ is allocation matrix of rudders, $v(t)=\left[v_{1}, v_{2}, v_{3}, v_{4}, v_{5}\right]^{T}$ is the control input vector, and $\delta(v(t))=\left[\delta_{a}, \delta_{e}, \delta_{r}, \delta_{y}, \delta_{z}\right]^{T}$ denotes the plant input vector subject to saturation nonlinearity which are ailerons, elevator, rudder, lateral deflection, and longitudinal deflection of the thrust vector control surface. The detailed expressions of corresponding terms in (19) can be found in [25].

The saturated function of $\delta_{i}\left(v_{i}\right)$ can be expressed as

$$
\delta_{i}\left(v_{i}\right)=\operatorname{sat}\left(v_{i}\right)= \begin{cases}\delta_{i M}, & v_{i}>\delta_{i M}, \\ v_{i}, & -\delta_{i M} \leq v_{i} \leq \delta_{i M}, \\ -\delta_{i M}, & v_{i}<-\delta_{i M},\end{cases}
$$

where $\delta_{i M}$ is the bound of $\delta_{i}\left(v_{i}\right), i=1,2,3,4,5, \delta(v)=$ $\left[\delta_{1}\left(v_{1}\right), \delta_{2}\left(v_{2}\right), \delta_{3}\left(v_{3}\right), \delta_{4}\left(v_{4}\right), \delta_{5}\left(v_{5}\right)\right]^{T}$.

Considering that the control effect of $\Omega$ is mainly determined by $g_{s} \omega$ and the effect of $g_{s \delta} \delta$ on slow-loop system is very small, $g_{s \delta} \delta$ is taken as a part of the compounded disturbance $D_{s}$. On the other hand, there exists modeling error for the NSV, and the NSV is affected by the unknown time-varying external disturbance in flight. Thus, (19) can be rewritten as

$$
\begin{gathered}
\dot{\Omega}=f_{s}+g_{s} \omega+D_{s}, \\
y_{s}=\Omega, \\
\dot{\omega}=f_{f}+g_{f} \delta(v)+D_{f}, \\
y_{f}=\omega,
\end{gathered}
$$

where $D_{s}=\Delta f_{s}+\Delta g_{s} \omega+g_{s \delta} \delta$ and $D_{f}=\Delta f_{f}+\Delta g_{f} \delta+d_{f}$ are the compounded disturbance of slowloop and fastloop, respectively; $\Delta f_{s}, \Delta g_{s}, \Delta f_{f}$, and $\Delta g_{f}$ are modeling error of slowloop and fastloop, $d_{f}$ is the time-varying external disturbance of fastloop, $g_{f}=g_{f 1} g_{f \delta}$.

In this paper, the control objective is that the robust attitude control is designed to render $y_{s}$ to follow the given desired trajectory $\Omega_{c}$ in the presence of the system uncertainty, unknown external disturbance, and input saturation. The external disturbance only acts on fast-loop system in the form of torque. On the other hand, the slow-loop system is only affected by the system uncertainty. The block diagram of the developed robust control scheme is shown in Figure 1.

To proceed the design of flight control scheme for the attitude motion (21) and (22) of the NSV, the following assumptions are required.

Assumption 7. For the attitude motion (21) and (22) of the NSV, the compounded disturbance of slow-loop system and its first-order derivative are bounded, that is, $\left|D_{s, i}\right| \leq \beta_{s, i}$, $\beta_{s, i}>0,\left|\dot{D}_{s, i}\right| \leq \beta_{d s, i}, \beta_{d s, i}>0, D_{s}=\left[D_{s, 1}, D_{s, 2}, D_{s, 3}\right]^{T}, \dot{D}_{s}$ $=\left[\dot{D}_{s, 1}, \dot{D}_{s, 2}, \dot{D}_{s, 3}\right]^{T}, \beta_{s}=\left[\beta_{s, 1}, \beta_{s, 2}, \beta_{s, 3}\right]^{T}, \beta_{d s}=\left[\beta_{d s, 1}, \beta_{d s, 2}\right.$, $\left.\beta_{d s, 3}\right]^{T}$. At the same time, the compounded disturbance of fastloop is bounded, that is, $\left|D_{f, i}\right| \leq \beta_{f, i}, \beta_{f, i}>0, D_{f}=$ $\left[D_{f, 1}, D_{f, 2}, D_{f, 3}\right]^{T}$.
Assumption 8 (see [22]). For the attitude motion (21) and (22) of the NSV, the desired attitude angle $\Omega_{c}$ and its second-order derivative are known, bounded, and continuous.

Assumption 9 (see [26]). For the attitude motion (21) and (22) of the NSV, generalized inverse matrices of the control gain matrices $g_{s}$ and $g_{f}$ exist.

Remark 10. For a practical system, the external disturbance is bounded since it actually exists. In addition, the parameter uncertainty $\Delta(\bullet)$ is the function of system state $\Omega$ or $\omega$. Thus, we assume that the boundaries of $D_{s}, \dot{D}_{s}$, and $D_{f}$ satisfy $\left|D_{s, i}\right| \leq \beta_{s, i},\left|\dot{D}_{s, i}\right| \leq \beta_{d s, i}$, and $\left|D_{f, i}\right| \leq \beta_{f, i}$, respectively. Accordingly, the Assumption 7 is reasonable.

3.2. Design of Slow-Loop System for the NSV. In slow-loop system, only the system uncertainty is considered. The adaptive control approach is used to estimate the upper bound of the system uncertainty. The robust control scheme of slowloop system is proposed based on the dynamic sliding mode control. To obtain the corresponding function's derivative, the higher-order sliding mode differentiator (HOSMD) [27-29] is employed because of the ability of its arbitrary approximation.

Theorem 11. Consider slow-loop system (21) of the NSV satisfied Assumptions 7-9. Under sliding modes (23) and (24), exponential reaching law (25), parameter updated law (26), and the dynamic sliding mode control law (27), the tracking error of slow-loop system asymptotically converges to the origin:

$$
\begin{gathered}
\sigma_{s 1}=e_{s}+\int_{0}^{t} A_{s 1} e_{s} d t, \\
\sigma_{s 2}=\dot{\sigma}_{s 1}+A_{s 2} \sigma_{s 1}, \\
\Phi_{\sigma_{s 2}}=-B_{s 1} \sigma_{s 2}-B_{s 2} \operatorname{sgn}\left(\sigma_{s 2}\right), \\
\dot{\hat{\beta}}_{d s}=B_{s 3}\left|\sigma_{s 2}\right|, \\
\omega_{c}=g_{s}^{-1} \int_{0}^{t}\left(\psi_{1}+\psi_{2}\right) d t, \\
\psi_{2}=-\dot{f}_{s}+\ddot{\Omega}_{c}-\left(A_{s 1}+A_{s 2}\right) \dot{e}_{s}-A_{s 2} A_{s 1} e_{s}, \\
B_{s 2} \operatorname{sgn}\left(\sigma_{s 2}\right)-\operatorname{diag}\left\{\operatorname{sgn}\left(\sigma_{s 2}\right)\right\} \hat{\beta}_{d s},
\end{gathered}
$$

where $e_{s}=\Omega-\Omega_{c}$ is the tracking error of slowloop, $A_{s 1}=$ $\operatorname{diag}\left\{a_{s 1,1}, a_{s 1,2}, a_{s 1,3}\right\}>0, A_{s 2}=\operatorname{diag}\left\{a_{s 2,1}, a_{s 2,2}, a_{s 2,3}\right\}>0$, $B_{s 1}=\operatorname{diag}\left\{b_{s 1,1}, b_{s 1,2}, b_{s 1,3}\right\}>0, B_{s 2}=\operatorname{diag}\left\{b_{s 2,1}, b_{s 2,2}, b_{s 2,3}\right\}>$ 0 , and $B_{s 3}=\operatorname{diag}\left\{b_{s 3,1}, b_{s 3,2}, b_{s 3,3}\right\}>0$ are designed matrices, and $\widehat{\beta}_{d s}$ is the estimate value of $\beta_{d s}$.

Proof. Consider the Lyapunov function as

$$
V_{s}=\frac{1}{2} \sigma_{s 2}^{T} \sigma_{s 2}+\frac{1}{2} \widetilde{\beta}_{d s}^{T} B_{s 3}^{-1} \widetilde{\beta}_{d s}
$$

where $\widetilde{\beta}_{d s}=\beta_{d s}-\widehat{\beta}_{d s}$ and $\dot{\tilde{\beta}}_{d s}=\dot{\beta}_{d s}-\dot{\hat{\beta}}_{d s}=-\dot{\hat{\beta}}_{d s}$. 
Differentiating (24) and considering (21), (23), and (27), we have

$$
\begin{aligned}
\dot{\sigma}_{s 2} & =\dot{f}_{s}+\psi_{1}+\psi_{2}+\dot{D}_{s}-\ddot{\Omega}_{c}+A_{s 1} \dot{e}_{s}+A_{s 2} \dot{e}_{s}+A_{s 2} A_{s 1} e_{s} \\
& =\dot{D}_{s}-B_{s 1} \sigma_{s 2}-B_{s 2} \operatorname{sgn}\left(\sigma_{s 2}\right)-\operatorname{diag}\left\{\operatorname{sgn}\left(\sigma_{s 2}\right)\right\} \widehat{\beta}_{d s} .
\end{aligned}
$$
by

Invoking (26) and (29), the time derivative of $V_{s}$ is given

$$
\begin{aligned}
\dot{V}_{s}= & \sigma_{s 2}^{T} \dot{\sigma}_{s 2}-\widetilde{\beta}_{d s}^{T} B_{s 3}^{-1} \dot{\hat{\beta}}_{d s} \\
= & \sigma_{s 2}^{T}\left(\dot{D}_{s}-B_{s 1} \sigma_{s 2}-B_{s 2} \operatorname{sgn}\left(\sigma_{s 2}\right)\right. \\
& \left.-\operatorname{diag}\left\{\operatorname{sgn}\left(\sigma_{s 2}\right)\right\} \widehat{\beta}_{d s}\right)-\widetilde{\beta}_{d s}^{T}\left|\sigma_{s 2}\right| \\
\leq & -\sigma_{s 2}^{T} B_{s 1} \sigma_{s 2}-\sigma_{s 2}^{T} B_{s 2} \operatorname{sgn}\left(\sigma_{s 2}\right)+\left|\sigma_{s 2}\right|^{T} \beta_{d s} \\
& -\left|\sigma_{s 2}\right|^{T} \widehat{\beta}_{d s}-\widetilde{\beta}_{d s}^{T}\left|\sigma_{s 2}\right| \\
= & -\sum_{i=1}^{3} b_{s 1, i} \sigma_{s 2, i}^{2}-\sum_{i=1}^{3} b_{s 2, i}\left|\sigma_{s 2, i}\right|,
\end{aligned}
$$

where $\sigma_{s 2}=\left[\sigma_{s 2,1}, \sigma_{s 2,2}, \sigma_{s 2,3}\right]$.

It is obvious that if $\sigma_{s 2} \neq 0$, then $\dot{V}_{s}<0$. Thus, the sliding mode $\sigma_{s 2}$ satisfies the reaching condition, and $\sigma_{s 2}$ can asymptotically converge to the origin. From (23) and (24), we can know that the sliding mode $\sigma_{s 1}$ and the tracking error $e_{s}$ converge to the origin when $\sigma_{s 2}$ converges to the origin. This concludes the proof.

Remark 12. $\omega_{c}$ in (27) is the controller of slow-loop system as well as the desired input of fast-loop system.

Remark 13. In the control law of the slow-loop system, the differential terms, such as $\dot{f}_{s}, \dot{e}_{s}$, and $\dot{\sigma}_{s 1}$, are difficult to obtain through derivative method. Thus, HOSMD is employed to get the corresponding derivatives. The form of $n$ th-order HOSMD is given by [27-29]

$$
\begin{aligned}
& \dot{z}_{0}=\varsigma_{0}=-\lambda_{0}\left|z_{0}-f(t)\right|^{n /(n+1)} \operatorname{sgn}\left(z_{0}-f(t)\right)+z_{1} \\
& \quad \vdots \\
& \dot{z}_{i}=\varsigma_{i}=-\lambda_{i}\left|z_{i}-\varsigma_{i-1}\right|^{(n-i) /(n-i+1)} \operatorname{sgn}\left(z_{i}-\varsigma_{i-1}\right)+z_{i+1} \\
& \quad \vdots \\
& \dot{z}_{n-1}=\varsigma_{n-1}=-\lambda_{n-1}\left|z_{n-1}-\varsigma_{n-2}\right|^{(1 / 2)} \operatorname{sgn}\left(z_{n-1}-\varsigma_{n-2}\right)+z_{n} \\
& \dot{z}_{n}=-\lambda_{n} \operatorname{sgn}\left(z_{n}-\varsigma_{n-1}\right),
\end{aligned}
$$

where $z_{i}$ and $\varsigma_{j}$ are states of the system (31), $\lambda_{0}, \lambda_{1}, \ldots, \lambda_{n}$ are designed parameters, and $f(t)$ is the known function. The aim of HOSMD is to make $\varsigma_{j}$ approximate the differential term $f(t)^{(j+1)}$ to arbitrary any accuracy, $i=0,1, \ldots, n, j=$ $0,1, \ldots, n-1$.
3.3. Design of Fast-Loop System for the NSV. In fast-loop system, the compounded disturbance is subjected to the system uncertainty as well as the unknown time-varying external disturbance. In general, the order of magnitude of the external disturbance is much larger than that of the system uncertainty. If we still use the adaptive scheme to handle the compounded disturbance of fast-loop system, the system trajectory will pass through the equilibrium point repeatedly. Thus, the designed disturbance cancellation can not accurately compensate the suffering disturbance and leads to the performance of the control system degeneracy. Here, the nonlinear disturbance observer is proposed to estimate the compounded disturbance. However, the robust control design of fast-loop system is different from that of slow-loop system based on DSMC and NDO. The reason is that the output derivative of NDO will be contained in the control law and it is more complex and difficult to prove the closed-loop system stability as well as the boundedness of disturbance observer error. Therefore, the traditional sliding mode control is used in fast-loop system, and the double power reaching law is designed to reduce the chattering of the system.

Considering that rudders are subject to saturation nonlinearity in fast-loop system and RBFNNs can smoothly approximate any continuous function over the compact set to arbitrary accuracy [30,31]. Here, RBFNNs are constructed as a compensator to overcome the saturation nonlinearity. Specifically, we employ RBFNNs to estimate the limited part exceeding the bound of saturation and use it to design the robust control law. As a result, the actuator can drop out the saturation nonlinearity.

Considering (20), we obtain

$$
\delta(v)=\operatorname{sat}(v)=v+\xi(v),
$$

where $\xi(v)$ is the limited part exceeding the actuator.

Using RBFNNs to approximate $\xi(v)$, we have

$$
\xi(v)=W_{\xi}^{* T} s_{\xi}(z)+\varepsilon_{\xi},
$$

where $W_{\xi}^{*} \in R^{l \times 5}$ are the optimal weights and satisfy $\left\|W_{\xi}^{*}\right\| \leq$ $W_{M}, l$ is the number of nodes; $z=\left[\omega_{c}, e_{f}\right]^{T}$ is the input vector of RBFNNs; $\varepsilon_{\xi}=\left[\varepsilon_{\xi, 1}, \varepsilon_{\xi, 2}, \varepsilon_{\xi, 3}, \varepsilon_{\xi, 4}, \varepsilon_{\xi, 5}\right]^{T}$ is the smallest approximated error, and $\varepsilon_{\xi}$ can be arbitrarily small through turning weights and nodes; suppose $\left|\varepsilon_{\xi, i}\right| \leq \varepsilon_{M \xi, i}$ and $\varepsilon_{M \xi, i}>0$ in this paper; $s_{\xi}(z)=\left[s_{\xi 1}, s_{\xi 2}, \ldots, s_{\xi l}\right]^{T}$ is the radial basis function vector, and $s_{\xi k}$ has the form as follows:

$$
s_{\xi k}=\exp \left(-\frac{\left\|z-c_{k}\right\|^{2}}{2 b_{k}^{2}}\right),
$$

where $c_{k}$ and $b_{k}$ are the center and width of the neural cell of the $k$ th hidden layer, $k=1,2, \ldots, l$.

Design the control law as

$$
v=v_{0}-v_{\xi}+v_{r},
$$

where $v_{0}$ is the controller by neglecting saturation nonlinearity, $v_{\xi}=\widehat{W}_{\xi}^{T} s_{\xi}(z)$ is the output of RBFNNs, and $v_{r}$ is the robust term. 
Substituting (33) and (35) into (32), we obtain

$$
\begin{aligned}
\delta(v) & =v_{0}-v_{\xi}+v_{r}+W_{\xi}^{* T} s_{\xi}(z)+\varepsilon_{\xi} \\
& =v_{0}+\widetilde{W}_{\xi}^{T} s_{\xi}(z)+v_{r}+\varepsilon_{\xi},
\end{aligned}
$$

where $\widetilde{W}_{\xi}=W_{\zeta}^{*}-\widehat{W}_{\xi}$

Considering the unknown compounded disturbance $D_{f}$ of the fast-loop system (22), it cannot be directly used in the design of fast-loop control scheme. To estimate the compounded disturbance, the nonlinear disturbance observer is presented below.

An auxiliary variable is given by

$$
\sigma=\eta-\omega
$$

And the derivative of $\eta$ is

$$
\dot{\eta}=-K \sigma-\operatorname{diag}\{\operatorname{sgn}(\sigma)\} \widehat{\beta}_{f}+f_{f}+g_{f} \delta(v)-K \sigma_{f},
$$

where $K=\operatorname{diag}\left\{k_{1}, k_{2}, k_{3}\right\}>0$ is a designed matrix, $\widehat{\beta}_{f}=\left[\widehat{\beta}_{f, 1}, \widehat{\beta}_{f, 2}, \widehat{\beta}_{f, 3}\right]^{T}$ is the estimate value of $\beta_{f}=$ $\left[\beta_{f, 1}, \beta_{f, 2}, \beta_{f, 3}\right]^{T}, \sigma=\left[\sigma_{1}, \sigma_{2}, \sigma_{3}\right]^{T}$ is the auxiliary variable, and $\sigma_{f}=\left[\sigma_{f, 1}, \sigma_{f, 2}, \sigma_{f, 3}\right]^{T}$ is the sliding mode surface of fastloop system, which will be introduced later.

The estimate value of $D_{f}$ can be written as

$$
\begin{aligned}
\widehat{D}_{f}= & -K \sigma-\operatorname{diag}\{\operatorname{sgn}(\sigma)\} \widehat{\beta}_{f}+\operatorname{diag}\left\{\operatorname{sgn}\left(\sigma_{f}\right)\right\}\left|\widehat{\beta}_{f}\right| \\
& +\operatorname{diag}\left\{\operatorname{sgn}\left(\sigma_{f}\right)\right\} \widehat{\beta}_{f} .
\end{aligned}
$$
have

Differentiating (37) and considering (22) and (38), we

$$
\dot{\sigma}=\dot{\eta}-\dot{\omega}=-K \sigma-\operatorname{diag}\{\operatorname{sgn}(\sigma)\} \widehat{\beta}_{f}-K \sigma_{f}-D_{f} .
$$

The disturbance approximation error is given by

$$
\widetilde{D}_{f}=D_{f}-\widehat{D}_{f}
$$

Substituting (39) and (40) into (41), we obtain

$$
\begin{aligned}
\widetilde{D}_{f}= & K \sigma+\operatorname{diag}\{\operatorname{sgn}(\sigma)\} \widehat{\beta}_{f} \\
& +D_{f}-\operatorname{diag}\left\{\operatorname{sgn}\left(\sigma_{f}\right)\right\}\left|\widehat{\beta}_{f}\right| \\
& -\operatorname{diag}\left\{\operatorname{sgn}\left(\sigma_{f}\right)\right\} \widehat{\beta}_{f} \\
= & -\dot{\sigma}-K \sigma_{f}-\operatorname{diag}\left\{\operatorname{sgn}\left(\sigma_{f}\right)\right\}\left|\widehat{\beta}_{f}\right| \\
& -\operatorname{diag}\left\{\operatorname{sgn}\left(\sigma_{f}\right)\right\} \widehat{\beta}_{f} .
\end{aligned}
$$

Theorem 14. Consider that fast-loop system (22) of the NSV satisfied Assumptions 7-9. The sliding mode is designed as (43), and the reaching law is chosen as (44). The nonlinear disturbance observer is proposed as (39), and the parameter adaptation law is designed as (45). RBFNNs are constructed as a compensator to overcome the saturation nonlinearity, and the updated law of the neural network weight is chosen as
(46). Under the control law (47), the tracking error of fast-loop system asymptotically converges to the origin, and the error of the disturbance observer also converges to the origin:

$$
\begin{gathered}
\sigma_{f}=e_{f}+\int_{0}^{t} A_{f} e_{f} d t, \\
\Phi_{\sigma_{f}}=-B_{f 1} \operatorname{diag}\left\{\operatorname{sgn}\left(\sigma_{f}\right)\right\}\left|\sigma_{f}\right|^{c_{1}} \\
-B_{f 2} \operatorname{diag}\left\{\operatorname{sgn}\left(\sigma_{f}\right)\right\}\left|\sigma_{f}\right|^{c_{2}}, \\
\dot{\hat{\beta}}_{f}=\Gamma_{\beta}\left(|\sigma|+\left|\sigma_{f}\right|\right), \\
\dot{\widehat{W}}_{\xi}=\Gamma_{\xi} s_{\xi}(z) \sigma_{f}^{T} g_{f}-\gamma|| \sigma_{f}^{T} g_{f}|| \Gamma_{\xi} \widehat{W}_{\xi}, \\
v=v_{0}-v_{\xi}+v_{r}, \\
v_{0}=g_{f}^{T}\left(g_{f} g_{f}^{T}\right)^{-1}\left(-f_{f}+\dot{\omega}_{c}-A_{f} e_{f}-\widehat{D}_{f}\right. \\
-B_{f 1} \operatorname{diag}\left\{\operatorname{sgn}\left(\sigma_{f}\right)\right\}\left|\sigma_{f}\right|^{c_{1}} \\
\left.-B_{f 2} \operatorname{diag}\left\{\operatorname{sgn}\left(\sigma_{f}\right)\right\}\left|\sigma_{f}\right|^{c_{2}}\right), \\
v_{\xi}=\widehat{W}_{\xi}^{T} s_{\xi}(z), \\
v_{r}=-K_{r} \operatorname{sgn}\left(g_{f}^{T} \sigma_{f}\right),
\end{gathered}
$$

where $e_{f}=\omega-\omega_{c}$ is the tracking error of fastloop; $A_{f}=$ $\operatorname{diag}\left\{a_{f, 1}, a_{f, 2}, a_{f, 3}\right\}>0, B_{f 1}=\operatorname{diag}\left\{b_{f 1,1}, b_{f 1,2}, b_{f 1,3}\right\}>0$, $B_{f 2}=\operatorname{diag}\left\{b_{f 2,1}, b_{f 2,2}, b_{f 2,3}\right\}>0, \Gamma_{\beta}=\Gamma_{\beta}^{T}>0, \Gamma_{\xi}=\Gamma_{\xi}^{T}>0$, and $K_{r}=\operatorname{diag}\left\{k_{r, 1}, k_{r, 2}, k_{r, 3}, k_{r, 4}, k_{r, 5}\right\}$ are designed matrices, and $k_{r, i}-\varepsilon_{M \xi, i}-W_{M}^{2} \gamma / 4>0 ; \gamma>0, c_{1}>1$ and $0<c_{2}<1$ are designed parameters.

Proof. Consider the Lyapunov function as

$$
V_{f}=\frac{1}{2} \sigma_{f}^{T} \sigma_{f}+\frac{1}{2} \sigma^{T} \sigma+\frac{1}{2} \widetilde{\beta}_{f}^{T} \Gamma_{\beta}^{-1} \widetilde{\beta}_{f}+\frac{1}{2} \operatorname{tr}\left(\widetilde{W}_{\xi}^{T} \Gamma_{\xi}^{-1} \widetilde{W}_{\xi}\right),
$$

where $\widetilde{\beta}_{f}=\beta_{f}-\widehat{\beta}_{f}, \widetilde{W}_{\xi}=W_{\xi}^{*}-\widehat{W}_{\xi}$, and $\dot{\tilde{\beta}}_{f}=\dot{\beta}_{f}-\dot{\hat{\beta}}_{f}=-\dot{\hat{\beta}}_{f}$ and $\dot{\widehat{W}}_{\xi}=\dot{W}_{\xi}^{*}-\dot{\widehat{W}}_{\xi}=-\dot{\widehat{W}}_{\xi}$.

Differentiating (43) and considering (22), we obtain

$$
\dot{\sigma}_{f}=f_{f}+g_{f} \delta(v)+D_{f}-\dot{\omega}_{c}+A_{f} e_{f}
$$

Substituting (36), (41), and (47) into (49), we have

$$
\begin{aligned}
\dot{\sigma}_{f}= & f_{f}+D_{f}-\dot{\omega}_{c}+A_{f} e_{f} \\
& +g_{f}\left(v_{0}+\widetilde{W}_{\xi}^{T} s_{\xi}(z)+v_{r}+\varepsilon_{\xi}\right) \\
= & \widetilde{D}_{f}-B_{f 1} \operatorname{diag}\left\{\operatorname{sgn}\left(\sigma_{f}\right)\right\}\left|\sigma_{f}\right|^{c_{1}} \\
& -B_{f 2} \operatorname{diag}\left\{\operatorname{sgn}\left(\sigma_{f}\right)\right\}\left|\sigma_{f}\right|^{c_{2}} \\
& +g_{f} \widetilde{W}_{\xi}^{T} s_{\xi}(z)-g_{f} K_{r} \operatorname{sgn}\left(g_{f}^{T} \sigma_{f}\right)+g_{f} \varepsilon_{\xi} .
\end{aligned}
$$



by

Invoking (40) and (50), the time derivative of $V_{f}$ is given

$$
\begin{aligned}
\dot{V}_{f}= & \sigma_{f}^{T} \dot{\sigma}_{f}+\sigma^{T} \dot{\sigma}-\widetilde{\beta}_{f}^{T} \Gamma_{\beta}^{-1} \dot{\hat{\beta}}_{f}-\operatorname{tr}\left(\widetilde{W}_{\xi}^{T} \Gamma_{\xi}^{-1} \dot{\widehat{W}}_{\xi}\right) \\
= & \sigma_{f}^{T} \widetilde{D}_{f}-\sum_{i=1}^{3} b_{f 1, i}\left|\sigma_{f, i}\right|^{1+c_{1}}-\sum_{i=1}^{3} b_{f 2, i}\left|\sigma_{f, i}\right|^{1+c_{2}} \\
& +\sigma_{f}^{T} g_{f} \widetilde{W}_{\xi}^{T} s_{\xi}(z)-\sigma_{f}^{T} g_{f} K_{r} \operatorname{sgn}\left(g_{f}^{T} \sigma_{f}\right) \\
& +\sigma_{f}^{T} g_{f} \mathcal{E}_{\xi}-\sum_{i=1}^{3} k_{i} \sigma_{i}^{2}-|\sigma|^{T} \widehat{\beta}_{f}-\sigma^{T} K \sigma_{f} \\
& -\sigma^{T} D_{f}-\widetilde{\beta}_{f}^{T} \Gamma_{\beta}^{-1} \dot{\hat{\beta}}_{f}-\operatorname{tr}\left(\widetilde{W}_{\xi}^{T} \Gamma_{\xi}^{-1} \dot{\widehat{W}}_{\xi}\right) .
\end{aligned}
$$

Substituting (52) into (51) yields

$$
\begin{aligned}
\dot{V}_{f} \leq & -\sum_{i=1}^{3} b_{f 1, i}\left|\sigma_{f, i}\right|^{1+c_{1}}-\sum_{i=1}^{3} b_{f 2, i}\left|\sigma_{f, i}\right|^{1+c_{2}} \\
& -\sum_{i=1}^{3} k_{i} \sigma_{i}^{2}-\sigma_{f}^{T} g_{f} K_{r} \operatorname{sgn}\left(g_{f}^{T} \sigma_{f}\right)+\sigma_{f}^{T} g_{f} \varepsilon_{\xi} \\
& +\left|\sigma_{f}\right|^{T} \widetilde{\beta}_{f}-|\sigma|^{T} \widehat{\beta}_{f}+|\sigma|^{T} \beta_{f}+\sigma_{f}^{T} g_{f} \widetilde{W}_{\xi}^{T} s_{\xi}(z) \\
& -\widetilde{\beta}_{f}^{T} \Gamma_{\beta}^{-1} \dot{\widehat{\beta}}_{f}-\operatorname{tr}\left(\widetilde{W}_{\xi}^{T} \Gamma_{\xi}^{-1} \dot{\widehat{W}}_{\xi}\right) \\
\leq & -\sum_{i=1}^{3} b_{f 1, i}\left|\sigma_{f, i}\right|^{1+c_{1}}-\sum_{i=1}^{3} b_{f 2, i}\left|\sigma_{f, i}\right|^{1+c_{2}} \\
& -\sum_{i=1}^{3} k_{i} \sigma_{i}^{2}-\sum_{i=1}^{3}\left(k_{r, i}-\varepsilon_{M \xi, i}\right)\left|\sigma_{f}^{T} g_{f, i}\right|+\left|\sigma_{f}\right|^{T} \widetilde{\beta}_{f} \\
& +|\sigma|^{T} \widetilde{\beta}_{f}-\widetilde{\beta}_{f}^{T} \Gamma_{\beta}^{-1} \dot{\hat{\beta}}_{f} \\
& -\operatorname{tr}\left(\widetilde{W}_{\xi}^{T} \Gamma_{\xi}^{-1} \dot{\widehat{W}}_{\xi}-\widetilde{W}_{\xi}^{T} s_{\xi}(z) \sigma_{f}^{T} g_{f}\right),
\end{aligned}
$$

where $g_{f, i}$ is the $i$ th column vector of the matrix $g_{f}$.
Considering parameter updated laws (45) and (46), we have

$$
\begin{aligned}
\dot{V}_{f} \leq & -\sum_{i=1}^{3} b_{f 1, i}\left|\sigma_{f, i}\right|^{1+c_{1}}-\sum_{i=1}^{3} b_{f 2, i}\left|\sigma_{f, i}\right|^{1+c_{2}}-\sum_{i=1}^{3} k_{i} \sigma_{i}^{2} \\
& -\sum_{i=1}^{3}\left(k_{r, i}-\varepsilon_{M \xi, i}\right)\left|\sigma_{f}^{T} g_{f, i}\right|+\operatorname{tr}\left(\gamma\left\|\sigma_{f}^{T} g_{f}\right\| \widetilde{W}_{\xi}^{T} \widehat{W}_{\xi}\right) .
\end{aligned}
$$

Considering the following fact that

$$
\operatorname{tr}\left(\widetilde{W}_{\xi}^{T}\left(W_{\xi}^{*}-\widetilde{W}_{\xi}\right)\right) \leq\left\|\widetilde{W}_{\xi}\right\|\left\|W_{\xi}^{*}\right\|-\left\|\widetilde{W}_{\xi}\right\|^{2}
$$

we have

$$
\begin{aligned}
\operatorname{tr}\left(\gamma\left\|\sigma_{f}^{T} g_{f}\right\| \widetilde{W}_{\xi}^{T} \widehat{W}_{\xi}\right) \\
\quad=\gamma\left\|\sigma_{f}^{T} g_{f}\right\| \operatorname{tr}\left(\widetilde{W}_{\xi}^{T}\left(W_{\xi}^{*}-\widetilde{W}_{\xi}\right)\right) \\
\quad \leq \gamma\left\|\sigma_{f}^{T} g_{f}\right\|\left(\left\|\widetilde{W}_{\xi}\right\| W_{M}-\left\|\widetilde{W}_{\xi}\right\|^{2}\right) \\
\quad=\gamma\left\|\sigma_{f}^{T} g_{f}\right\|\left(-\left(\left\|\widetilde{W}_{\xi}\right\|-\frac{1}{2} W_{M}\right)^{2}+\frac{1}{4} W_{M}^{2}\right) .
\end{aligned}
$$

Substituting (56) into (54) yields

$$
\dot{V}_{f} \leq-\sum_{i=1}^{3} b_{f 1, i}\left|\sigma_{f, i}\right|^{1+c_{1}}-\sum_{i=1}^{3} b_{f 2, i}\left|\sigma_{f, i}\right|^{1+c_{2}}
$$

$$
\begin{aligned}
& -\sum_{i=1}^{3} k_{i} \sigma_{i}^{2}-\sum_{i=1}^{3}\left(k_{r, i}-\varepsilon_{M \xi, i}\right)\left|\sigma_{f}^{T} g_{f, i}\right| \\
& +\frac{1}{4} W_{M}^{2} \gamma\left\|\sigma_{f}^{T} g_{f}\right\|-\gamma\left\|\sigma_{f}^{T} g_{f}\right\|\left(\left\|\widetilde{W}_{\xi}\right\|-\frac{1}{2} W_{M}\right)^{2} \\
\leq & -\sum_{i=1}^{3} b_{f 1, i}\left|\sigma_{f, i}\right|^{1+c_{1}}-\sum_{i=1}^{3} b_{f 2, i}\left|\sigma_{f, i}\right|^{1+c_{2}} \\
& -\sum_{i=1}^{3} k_{i} \sigma_{i}^{2}-\sum_{i=1}^{3}\left(k_{r, i}-\varepsilon_{M \xi, i}-\frac{1}{4} W_{M}^{2} \gamma\right)\left|\sigma_{f}^{T} g_{f, i}\right| \\
& -\gamma\left\|\sigma_{f}^{T} g_{f}\right\|\left(\left\|\widetilde{W}_{\xi}\right\|-\frac{1}{2} W_{M}\right)^{2} .
\end{aligned}
$$

If $k_{r, i}-\varepsilon_{M \xi, i}-W_{M}^{2} \gamma / 4>0$, then $\dot{V}_{f}<0$. Thus, $\sigma_{f}$ and $\sigma$ converge to the origin. According to (43), we obtain that the tracking error $e_{f}$ converges to the origin. When $\sigma$ converges to the origin, we have $\dot{\sigma}=0$. Thus, we can know that the approximation error $\widetilde{D}_{f}$ converges to the origin from (42). This includes the proof.

Remark 15. For the double power reaching law [32-34], the first term of (44) plays a key role when the system state is far away from the sliding mode $\left(\left|\sigma_{f, i}\right|>1\right)$, and the speed of the 


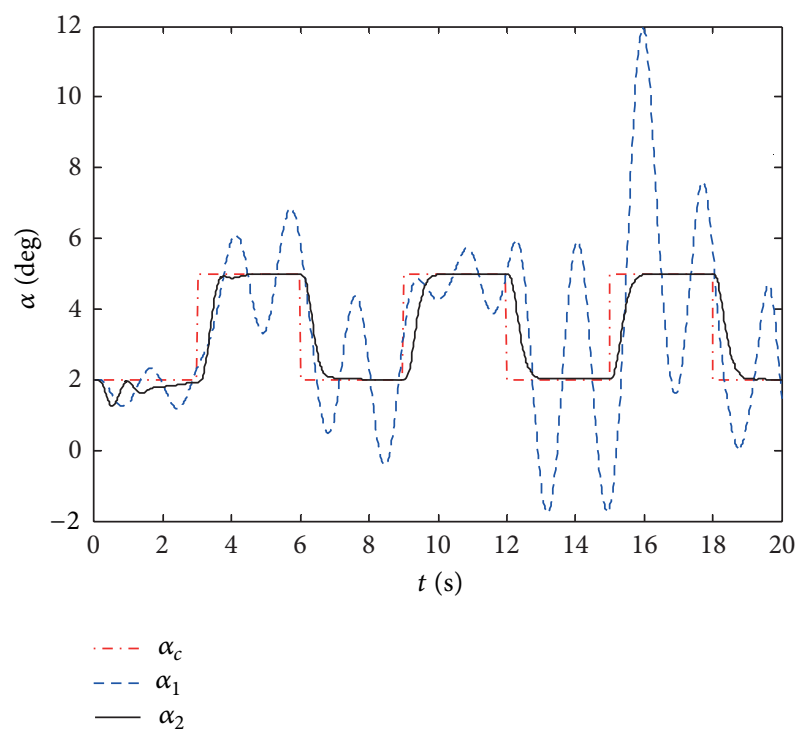

Figure 2: Angle of attack.

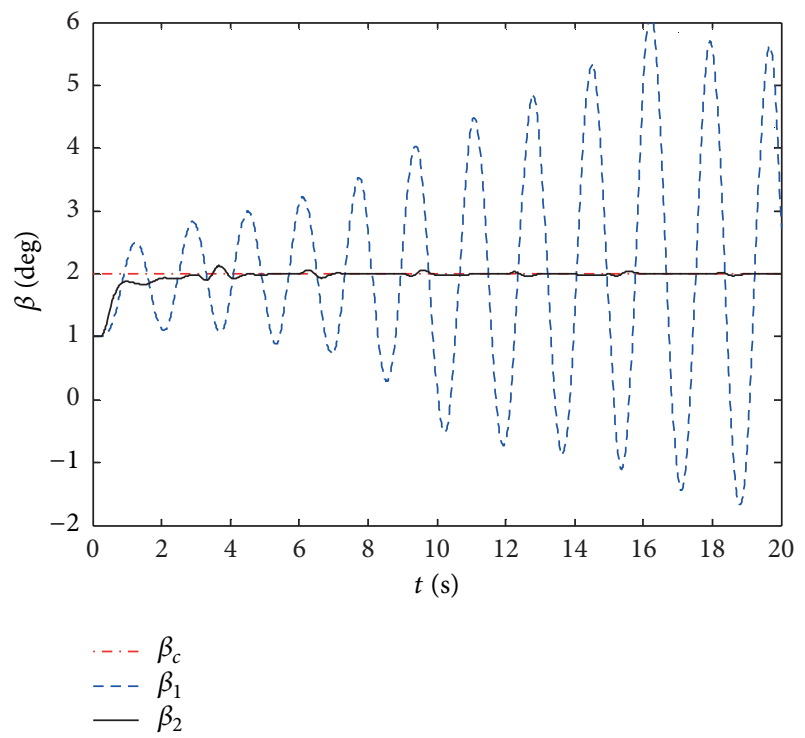

FIGURE 3: Sideslip angle.

double power reaching law is faster than that of the standard reaching law. On the other hand, when the system state is close to the sliding mode $\left(\left|\sigma_{f, i}\right|<1\right)$, the second term of (44) plays a key role, and the speed of the double power reaching law is lower than that of the standard reaching law. Therefore, the chattering phenomenon can be attenuated through the double power reaching law.

3.4. Simulation Study for the NSV. In this section, the simulation results are given to illustrate the effectiveness of the proposed attitude control schemes for the NSV. The initial values are chosen as $\alpha_{0}=2^{\circ}, \beta_{0}=1^{\circ}, \mu_{0}=0^{\circ}, p_{0}=q_{0}=r_{0}=$ $0 \mathrm{rad} / \mathrm{s}, H_{0}=22000 \mathrm{~m}, V_{0}=600 \mathrm{~m} / \mathrm{s}, \delta_{a M}=\delta_{e M}=\delta_{r M}=$ $30^{\circ}$, and $\delta_{y M}=\delta_{z M}=15^{\circ}$. The desired flight attitudes are

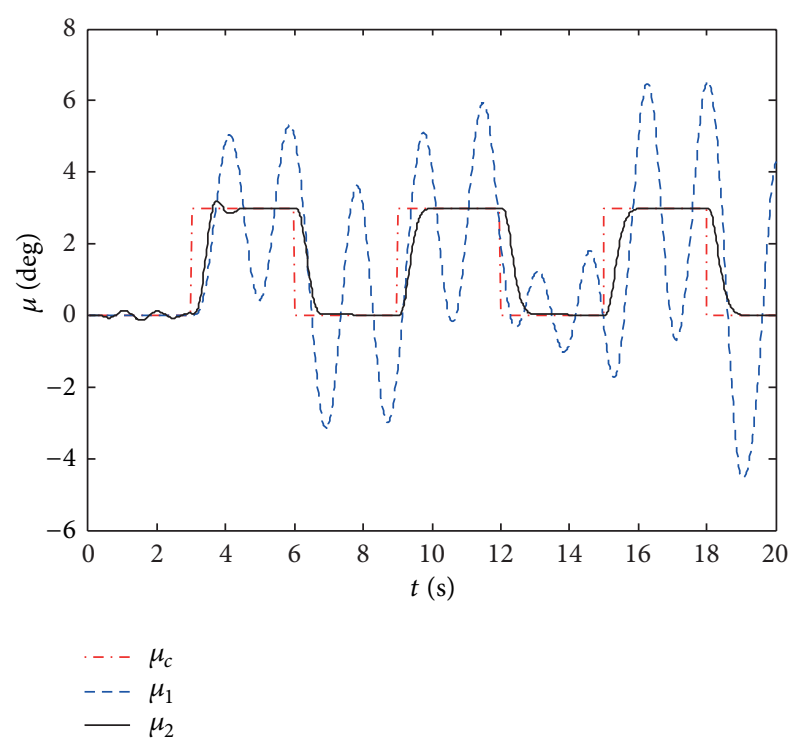

Figure 4: Roll angle.

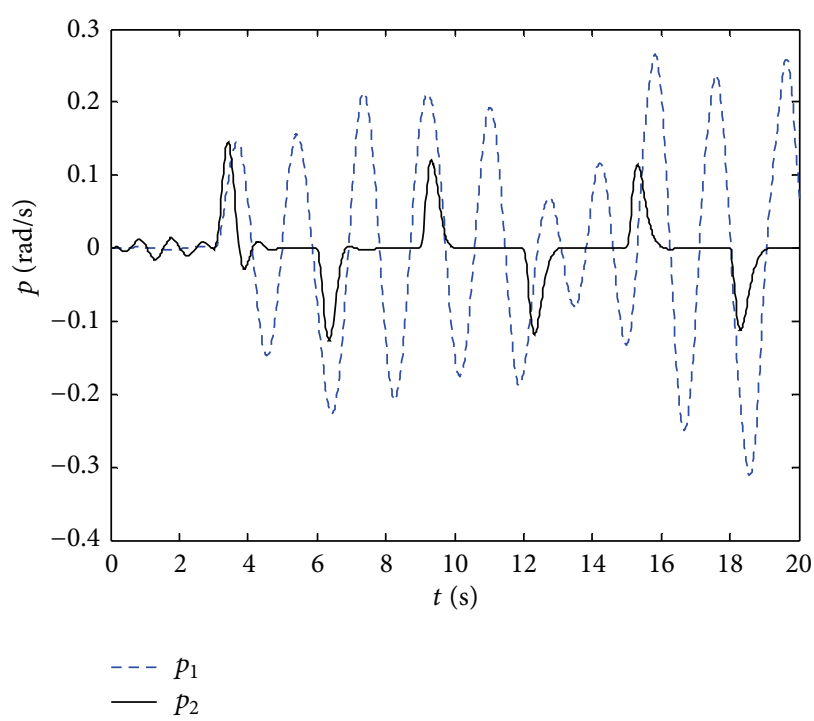

FIgURE 5: Roll angular rate.

chosen as

$$
\begin{gathered}
\alpha_{c}= \begin{cases}2^{\circ}, & 3 k \leq t<3(k+1), \\
5^{\circ}, & 3(k+1) \leq t<3(k+2),\end{cases} \\
\beta_{c}=2^{\circ}, \\
\mu_{c}= \begin{cases}0^{\circ}, & 3 k \leq t<3(k+1), \\
3^{\circ}, & 3(k+1) \leq t<3(k+2), \\
k=0,1,2, \ldots\end{cases}
\end{gathered}
$$

Suppose that there are $+20 \%$ and $-20 \%$ uncertainties on aerodynamic coefficients and aerodynamic moment coefficients, respectively. On the other hand, the unknown external 


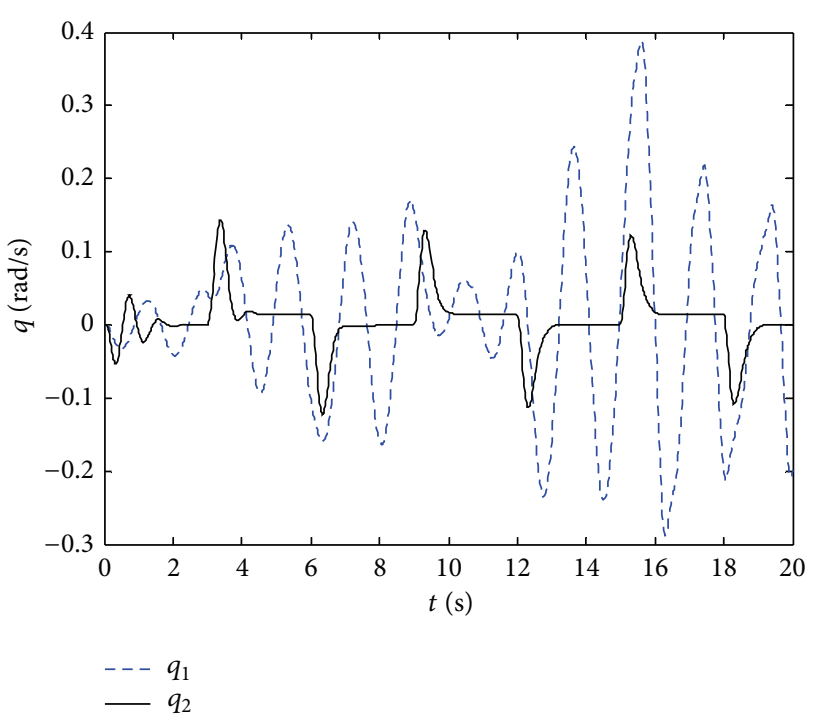

FIGURE 6: Pitch angular rate.

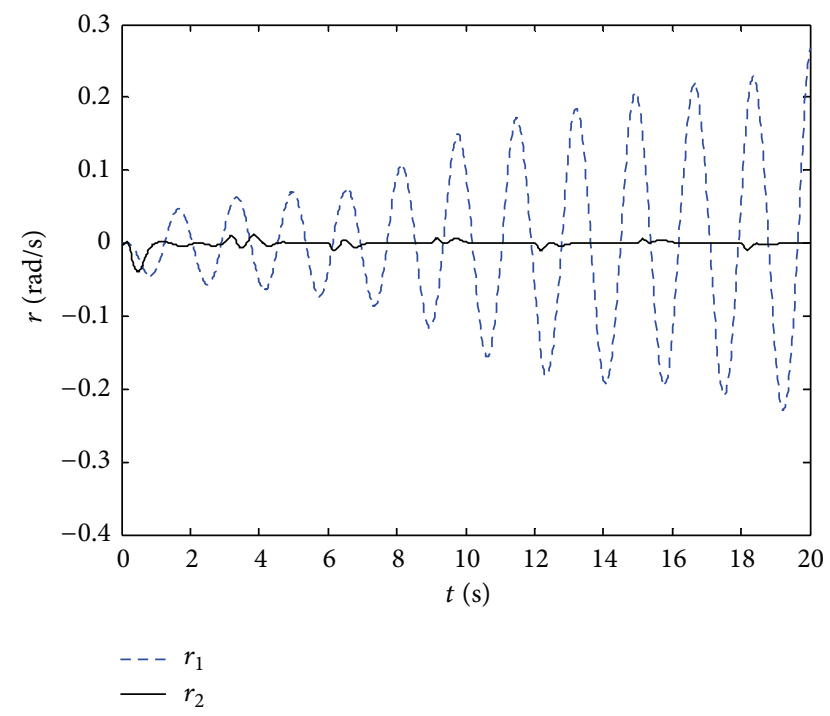

FIGURE 7: Yaw angular rate.

disturbance moments in fast-loop system of the NSV are given by

$$
\begin{gathered}
d_{M f, 1}(t)=4 \times 10^{5}(\sin (5 t)+0.2) N m, \\
d_{M f, 2}(t)=4 \times 10^{6}(\cos (5 t)+0.1) N m, \\
d_{M f, 3}(t)=4 \times 10^{6} \sin (5 t) N m .
\end{gathered}
$$

In order to obtain continuous and bounded derivatives of the desired flight attitudes, two-order reference model $G(s)=\omega_{s}^{2} /\left(s^{2}+2 \xi \omega_{s} s+\omega_{s}^{2}\right)$ is employed, where $\omega_{s}$ and $\xi$ are designed parameters. All parameters are given as $A_{s 1}=$ $\operatorname{diag}\{1.5,1,1\}, A_{s 2}=\operatorname{diag}\{1,1,1\}, B_{s 1}=\operatorname{diag}\{0.2,0.2,0.2\}$,

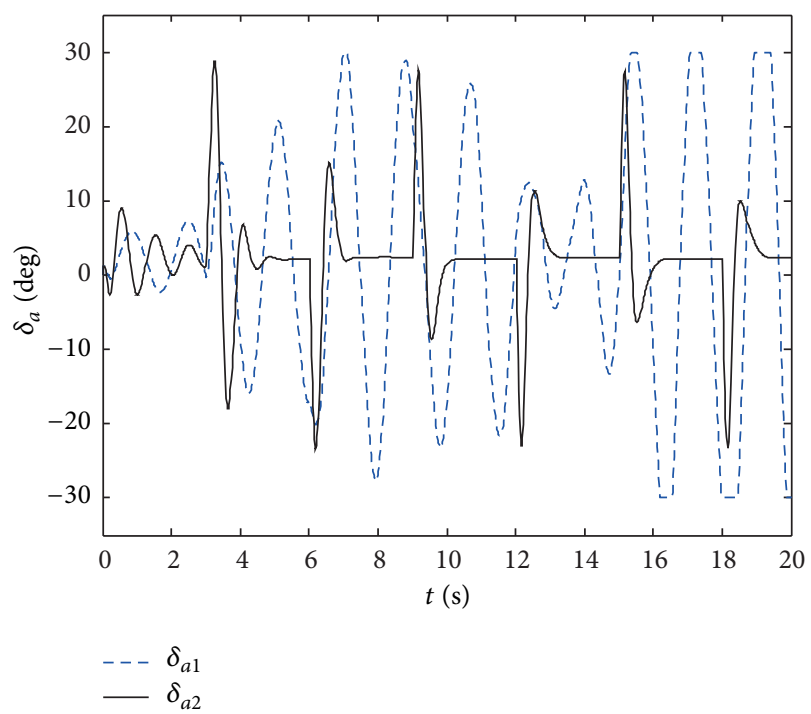

FIGURE 8: Ailerons.

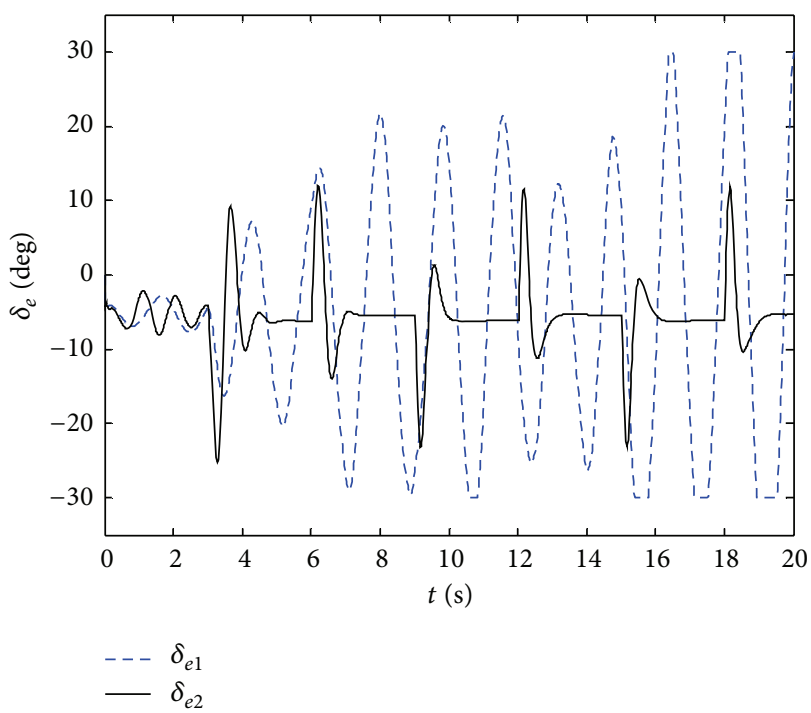

FIGURE 9: Elevator.

$B_{s 2}=\operatorname{diag}\{0.2,0.2,0.2\}, B_{s 3}=\operatorname{diag}\{0.1,0.1,0.1\}, A_{f}=$ $\operatorname{diag}\{0.5,0.5,0.5\}, B_{f 1}=\operatorname{diag}\{0.18,0.18,0.18\}, B_{f 2}=$ $\operatorname{diag}\{0.18,0.18,0.18\}, K=\operatorname{diag}\{6,6,6\}, \Gamma_{\beta}=\operatorname{diag}\{4,4,4\}$, $\Gamma_{\xi}=\operatorname{diag}\{50\}_{15 \times 15}, K_{r}=\operatorname{diag}\{20,20,20,20,20\}, \gamma=0.001$, $c_{1}=2.5, c_{2}=0.5, \omega_{s}=4$, and $\xi=0.8$.

The simulation results are shown in Figures 2, 3, 4, 5, 6, 7, $8,9,10,11$, and 12 , where dash dot lines (with subscript " $c$ ") represent the desired attitudes and dot lines (with subscript "1") stand for the responses without NDO and saturation compensation, while solid lines (with subscript "2") represent the responses with NDO and saturation compensation.

From Figures 2 to 4, we can observe that the small tracking errors of attitude angles are obtained and the attitude angular rates tend to be stable from Figures 5 to 7 based on the proposed sliding mode control scheme while the oscillation phenomena emerge without the nonlinear 


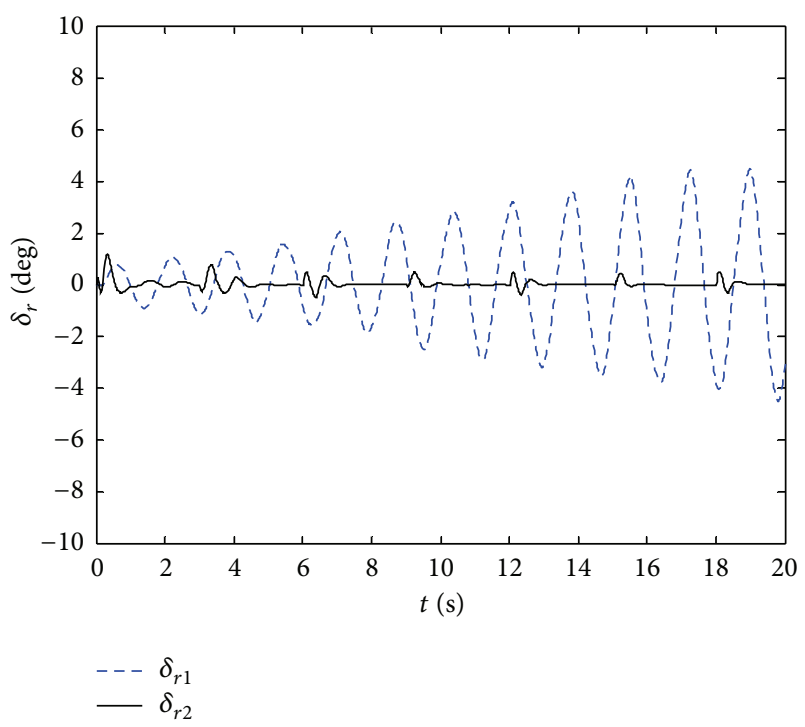

FIGURE 10: Rudder.

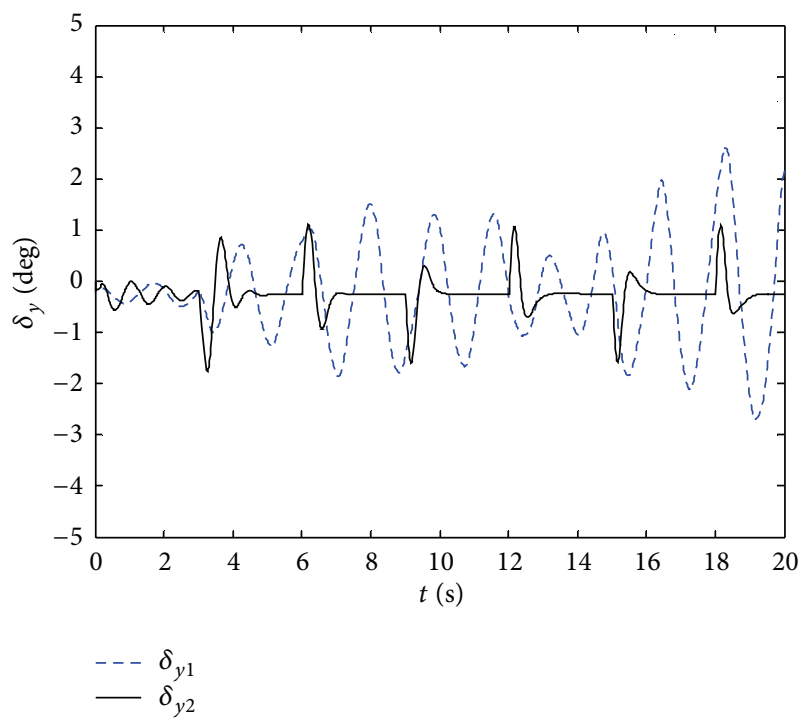

FIGURE 11: Lateral deflection of the thrust vectoring control surface.

disturbance observer and RBFNNs saturation compensator. On the other hand, the control inputs are presented from Figures 8 to 12 . Under the proposed robust control scheme, it is clear that all deflections of rudders are in the saturation range. The deflections of ailerons and elevator reach the saturation limits without the nonlinear disturbance observer and RBFNNs saturation compensator. That is, the developed robust control scheme for the NSV can prevent the control signals from reaching saturation limits. From the previously stated analysis, the satisfactory attitude tracking control performance is obtained under the proposed robust control scheme for the NSV in the presence of the unknown external disturbance and the input saturation. Thus, the proposed robust control scheme is valid for the NSV.

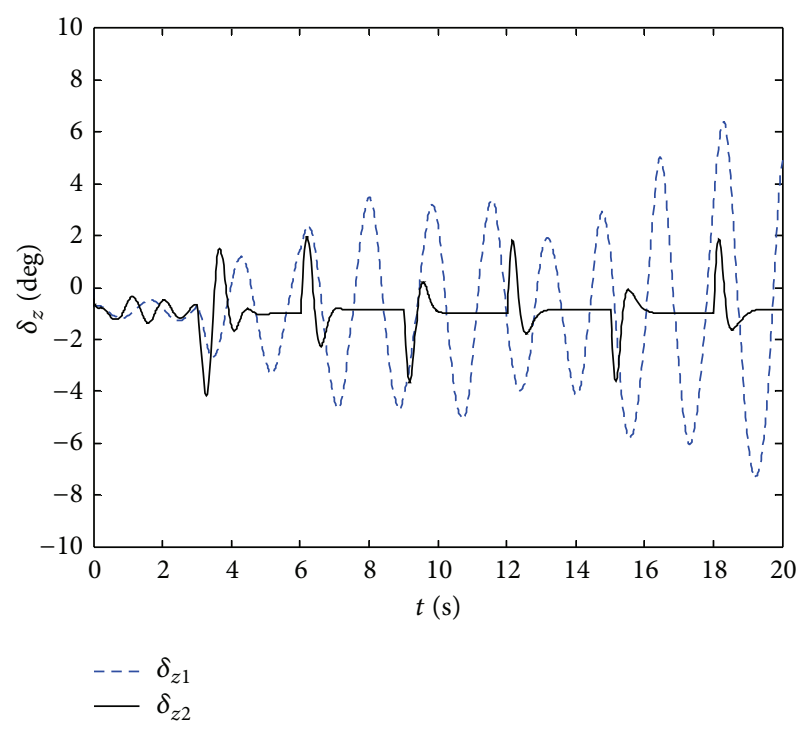

FIGURE 12: Longitudinal deflection of the thrust vectoring control surface.

\section{Conclusion}

An effective control scheme is proposed for the NSV with strong nonlinearity, high coupling, parameter uncertainty, and unknown time-varying disturbance based on SMC and NDO. Firstly, the nonlinear disturbance observer is designed to handle the compounded disturbance. Secondly, considering saturation characteristic of rudders, RBFNNs are constructed as a compensator to overcome the saturation nonlinearity. The stability of the closed-loop system is proved, and the tracking error as well as the disturbance observer error can converge to the origin through the Lyapunov analysis. Finally, Simulation results demonstrate the effectiveness of the proposed flight control scheme.

\section{Acknowledgments}

This work is partially supported by the National Natural Science Foundation of China (Grant no. 61174102), Jiangsu Natural Science Foundation of China (Grant no. SBK2011069), Program for New Century Excellent Talents in University of China (Granted no. NCET-11-0830), NUAA Research Funding (Grant no. NS2013028), and "Liu Da Ren Cai Gao Feng" scheme of Jiangsu Province in China (Granted no. 2012-XXRJ-010).

\section{References}

[1] Y. G. Wang, J. Q. Li, Y. Li, and W. Yao, "Characters and application prospects of near space flying vehicles," Spacecraft Engineering, vol. 16, no. 1, pp. 50-57, 2007.

[2] E. J. Cui, "Research statutes, development trends and key technical problems of near space flying vehicles," Advances Mechanics, vol. 39, no. 6, pp. 658-673, 2009.

[3] W. S. Nie, S. B. Luo, S. J. Feng, and F. C. Zhuang, "Analysis of key technologies and development trend of near space vehicle," 
Journal of National University of Defense Technology, vol. 34, no. 2, pp. 107-113, 2012.

[4] M. Chen and B. Jiang, "Robust attitude control of near space vehicles with time-varying disturbances," International Journal of Control, Automation, and Systems, vol. 11, no. 1, pp. 182-187, 2013.

[5] M. Chen and W.-H. Chen, "Sliding mode control for a class of uncertain nonlinear system based on disturbance observer," International Journal of Adaptive Control and Signal Processing, vol. 24, no. 1, pp. 51-64, 2010.

[6] J. H. Wang, J. B. Hu, and B. F. Zhang, "Backstepping terminal sliding mode for flight control based on nonlinear disturbance observer," Journal of Applied Sciences, vol. 30, no. 4, pp. 408-414, 2012.

[7] M. Chen, R. Mei, and B. Jiang, "Sliding mode control for a class of uncertain MIMO nonlinear systems with application to nearspace vehicles," Mathematical Problems in Engineering, vol. 2013, Article ID 180589, 9 pages, 2013.

[8] Y. H. Luo, H.-G. Zhang, and D.-S. Yang, "Neural network controller design and simulation based on saturation compensator," Journal of System Simulation, vol. 21, no. 1, pp. 184-188, 2009.

[9] W. Z. Gao, Intelligent Control of Nonlinear Systems with Actuator Saturation Using Neural Networks, Louisiana Tech University, 2005.

[10] B. Xu, Design of Adaptive Controller for a Class of Uncertain Nonlinear Systems with Input Saturation, China University of Petroleum, 2010.

[11] C. Wen, J. Zhou, Z. Liu, and H. Su, "Robust adaptive control of uncertain nonlinear systems in the presence of input saturation and external disturbance," IEEE Transactions on Automatic Control, vol. 56, no. 7, pp. 1672-1678, 2011.

[12] Y. M. Li, S. C. Tong, and T. S. Li, "Direct adaptive fuzzy backstepping control of uncertain nonlinear systems in the presence of input saturation," Neural Computing and Applications, 2012.

[13] Z. Li and Y. Zhang, "Robust adaptive motion/force control for wheeled inverted pendulums," Automatica, vol. 46, no. 8, pp. 1346-1353, 2010.

[14] D. Qian, J. Yi, and D. Zhao, "Robust control using sliding mode for a class of under-actuated systems with mismatched uncertainties," in Proceedings of IEEE International Conference on Robotics and Automation (ICRA '07), pp. 1449-1454, Roma, Italy, April 2007.

[15] Z. Gao, B. Jiang, H. Gong, and Y. Xu, "Fault-tolerant sliding mode control design for near space vehicle based on T-S fuzzy model," in Proceedings of the 4th International Conference on Innovative Computing, Information and Control (ICICIC '09), pp. 211-214, December 2009.

[16] Z. Li, J. Li, and Y. Kang, "Adaptive robust coordinated control of multiple mobile manipulators interacting with rigid environments," Automatica, vol. 46, no. 12, pp. 2028-2034, 2010.

[17] C. J. Schumacher, G. C. Cottrill, and H. H. Yeh, "Optimal sliding mode flight control," in AIAA Guidance, Navigation and Control Conference and Exhibit, pp. 1-9, AIAA, Portlad, Or, USA, 1999.

[18] Z. Li and Y. Kang, "Dynamic coupling switching control incorporating Support Vector Machines for wheeled mobile manipulators with hybrid joints," Automatica, vol. 46, no. 5, pp. 832-842, 2010.

[19] J. K. Liu, MATLAB Simulation for Sliding Mode Control, University Press, Beijing, China, 2005.

[20] J.-Y. Kuan and H.-P. Huang, "Independent joint dynamic sliding mode control of a humanoid robot arm," in Proceedings of IEEE
International Conference on Robotics and Biomimetics (ROBIO '08), pp. 602-607, Bangkok, Thailand, February 2009.

[21] A. J. Koshkouei, K. J. Burnham, and A. S. I. Zinober, "Dynamic sliding mode control design," IEE Proceedings: Control Theory and Applications, vol. 152, no. 4, pp. 392-396, 2005.

[22] M. Pu, Q.-X. Wu, C.-S. Jiang, and J. Zhang, "Near space vehicle control based on second-order dynamic Terminal sliding mode," Journal of Astronautics, vol. 31, no. 4, pp. 1056-1062, 2010.

[23] M. Pu, Q. Wu, C. Jiang, and L. Cheng, "Application of secondorder dynamic Terminal sliding mode control based on nonlinear disturbance observer to near space vehicle," Journal of Southeast University, vol. 39, no. 1, pp. 68-75, 2009.

[24] A. Isidori, Nonlinear Control Systems. II, Communications and Control Engineering Series, Springer, London, UK, 1999.

[25] J. Pang, R. Mei, and M. Chen, "Modeling and control for near space vehicles with oblique wing," in Proceedings of the 10th World Congress on Intelligent Control and Automation, pp. 17731778, Beijing, China, 2012.

[26] C. Yang, Y. Li, S. S. Ge, and T. H. Lee, "Adaptive control of a class of discrete-time MIMO nonlinear systems with uncertain couplings," International Journal of Control, vol. 83, no. 10, pp. 2120-2133, 2010.

[27] A. Levant, "Higher-order sliding modes, differentiation and output-feedback control," International Journal of Control, vol. 76, no. 9-10, pp. 924-941, 2003.

[28] J.-S. Fan, H.-X. Zhang, G.-M. Wang, and X.-G. Wang, "Improvement of higher order sliding mode control and its application," Control and Decision, vol. 26, no. 9, pp. 1436-1440, 2011.

[29] B. L. Tian, W. R. Fan, Q. Zong, J. Wang, and F. Wang, "Adaptive high order sliding mode controller design for hypersonic vehicle with flexible body dynamics," Mathematical Problems in Engineering, vol. 2013, Article ID 357685, 11 pages, 2013.

[30] C. Yang, S. S. Ge, C. Xiang, T. Chai, and T. H. Lee, "Output feedback NN control for two classes of discrete-time systems with unknown control directions in a unified approach," IEEE Transactions on Neural Networks, vol. 19, no. 11, pp. 1873-1886, 2008.

[31] Z. Li and C. Yang, "Neural-adaptive output feedback control of a class of transportation vehicles based on wheeled inverted pendulum models," IEEE Transactions on Control Systems Technology, vol. 20, no. 6, pp. 1583-1591, 2012.

[32] L. P. Xi, Z. L. Chen, and S. H. Zhang, "Design of sliding mode control scheme based on improved idempotent trending law for robotic manipulators," Computer Measurement \& Control, vol. 20, no. 2, pp. 380-382, 2012.

[33] H. Mei and Y. Wang, "Fast convergent sliding mode variable structure control of robot," Information and Control, vol. 38, no. 5, pp. 552-557, 2009.

[34] H. X. Zhang, J. S. Fan, F. Meng, and J. F. Huang, "A new double power reaching law for sliding mode control," Control and Decision, vol. 28, no. 2, pp. 289-293, 2013. 


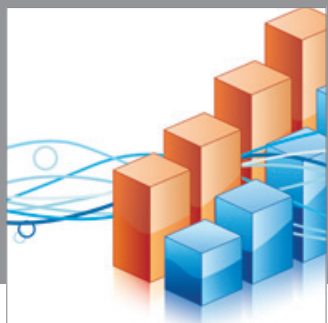

Advances in

Operations Research

mansans

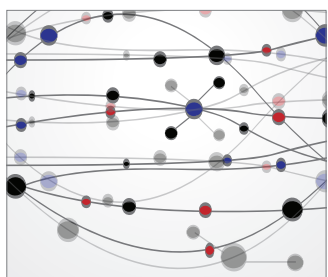

The Scientific World Journal
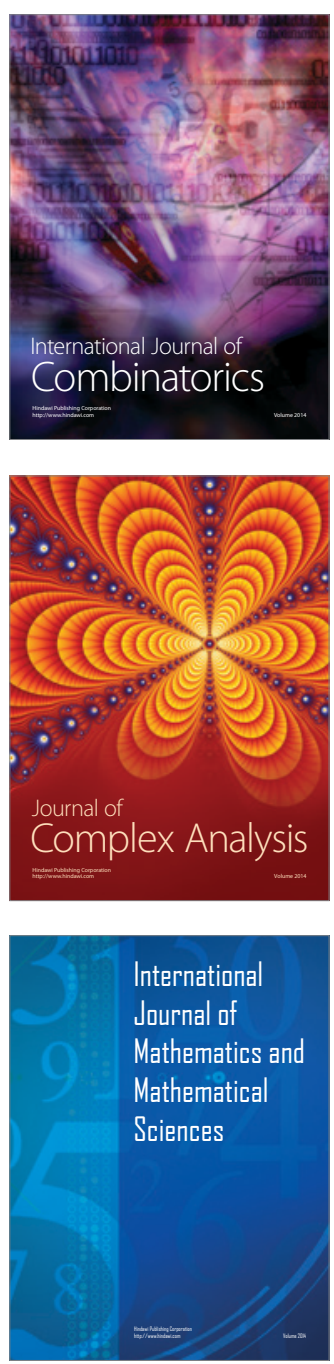
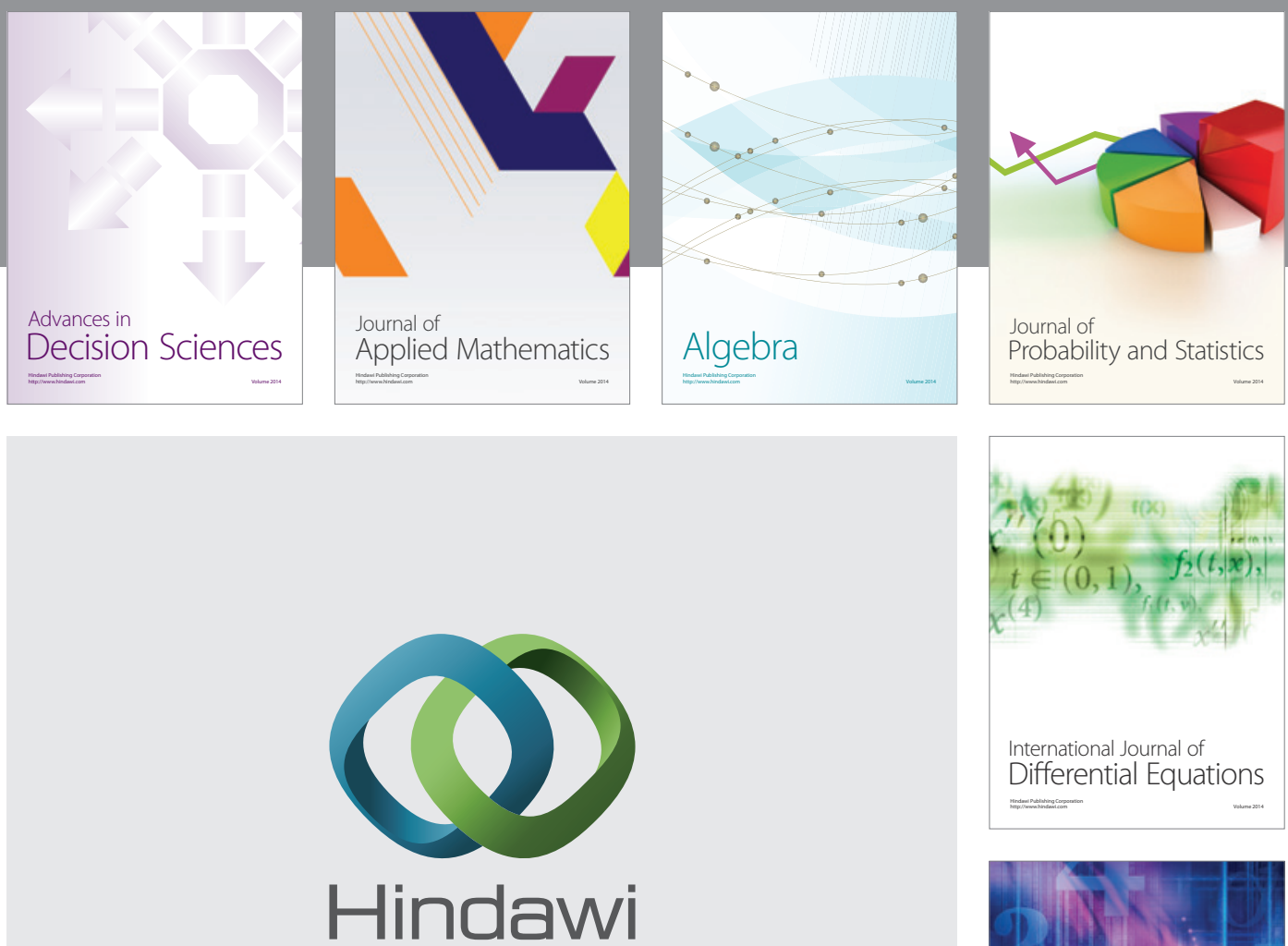

Submit your manuscripts at http://www.hindawi.com
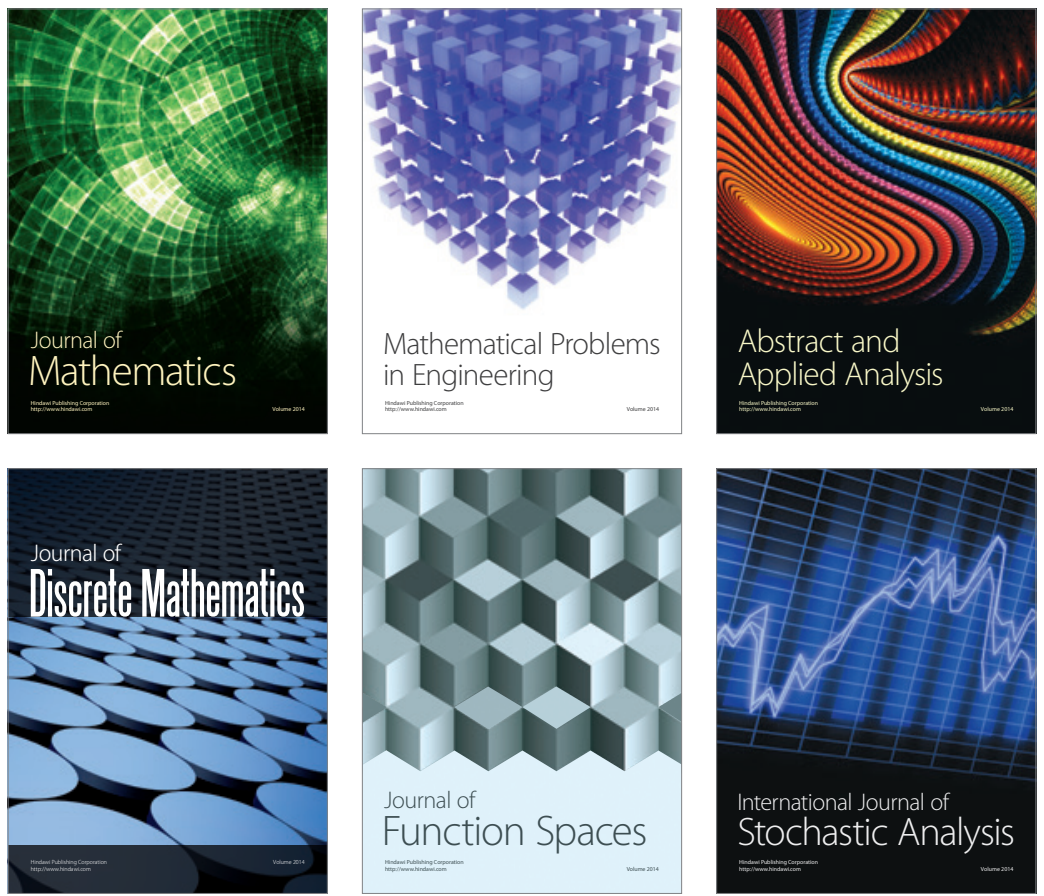

Journal of

Function Spaces

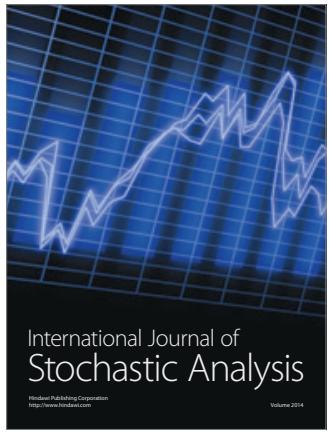

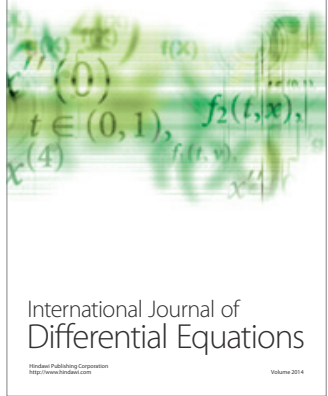
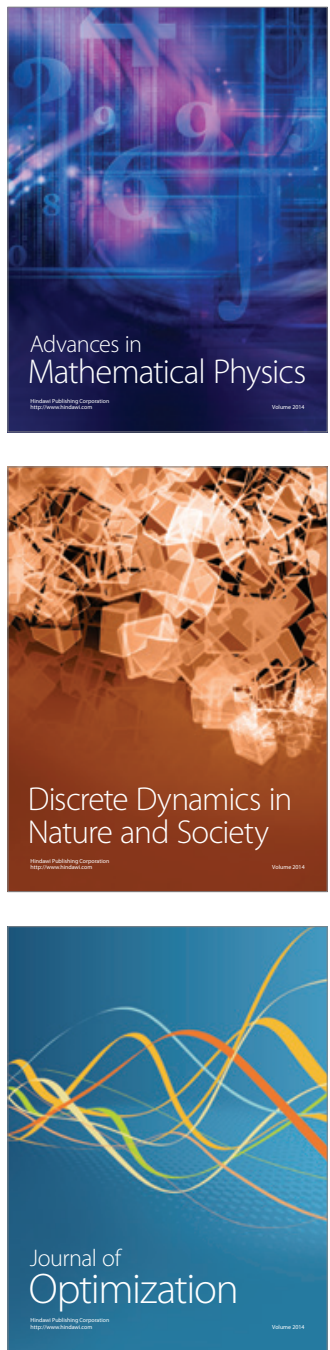